\title{
Remarques sur les critiques chinoises du Contrat social
}

Albert Chen Lichuan

\section{(2) OpenEdition}

Journals

Édition électronique

URL : http://journals.openedition.org/transtexts/92

DOI : 10.4000/transtexts.92

ISSN : 2105-2549

Éditeur

Gregory B. Lee

Édition imprimée

Date de publication : 1 janvier 2007

Pagination : 155-182

ISSN : 1771-2084

\section{Référence électronique}

Albert Chen Lichuan, «Remarques sur les critiques chinoises du Contrat social », Transtext(e)s

Transcultures 跨文本跨文化 [En ligne], 2 | 2007, mis en ligne le 11 juin 2009, consulté le 19 avril 2019.

URL : http://journals.openedition.org/transtexts/92 ; DOI : 10.4000/transtexts.92 


\title{
Remarques sur les critiques chinoises du Contrat social
}

\author{
Albert Chen Lichuan
}

Within the history of political thought in twentieth-century China, critical literature on The Social Contract has thrived during two periods. At the turn of the century, intellectuals mainly debated Rousseau's apriorism and the utopian view of his book. Intellectuals from the last two decades of the century focused on the book's consequences on political practice and on its intrinsic link to totalitarianism. However, they failed to emphasize that the totalitarian regime of a single party is by definition contrary to the Rousseauist conception of "popular sovereignty" and of a Republic ruled by law.

Si l'on voulait trouver un ouvrage français de philosophie politique qui ait suscité en Chine autant d'admiration que de ressentiment chez les intellectuels de premier rang, c'est sans doute le Contrat Social de Jean-Jacques Rousseau qui s'imposerait. ${ }^{1}$

${ }^{1}$ La première traduction chinoise du Contrat Social est une retraduction de Nakae Tokusuke 中江篤介,
traducteur japonais de Rousseau au XIXe siècle. Il s'agit du Livre premier de cet ouvrage, publié à
Shanghai en 1898, l'année de la Réforme des Cent Jours, sous le titre de Minyue tongyi 民約通義. En 1902
paraît la première traduction intégrale de Yang Tingdong 楊廷棟, intitulée Lusuo minyuelun 路索民約論.
En 1918, Ma Junwu 馬君武 fait éditer à Pékin une autre traduction sous le titre de Lusuo minyuelun 盧騷
民約論. En 1938 paraît une traduction à Pékin de Xu Baiqi 徐百齐 et Qiujin 丘瑾, ayant pour titre
sheyuelun 社約論. En 1958, He Zhaowu 何兆武 publie sa traduction du Contrat Social, titrée Minyuelun
民約論. Entre 1963 et 2003, la traduction de He Zhaowu est rééditée douze fois par Shangwu yinshuguan 
Comment peut-on expliquer la réception de cet ouvrage pour le moins paradoxale dans l'histoire intellectuelle chinoise du XXe siècle? S'agit-il d'incompréhension, d'interprétation erronée ou de critiques sinon justes, du moins justifiées? Comment mesurer l'impact de cet ouvrage dans la formation de la conscience citoyenne des Chinois, notamment à travers le concept de «la souveraineté du peuple»? Dans quelle mesure $\mathrm{Du}$ Contrat social a-t-il servi de ferment aux réflexions sur le projet institutionnel que mènent les intellectuels chinois d'aujourd'hui? Sans prétendre répondre par cette modeste étude à toutes les questions posées ici, nous nous estimerions heureux d'y apporter quelques prémices de réponse.

\section{La critique du Contrat social au XXe siècle}

La critique du Contrat social en Chine commence vraisemblablement avec Yan Fu 嚴復 qui publie en février 1914 dans le Yongyan Bao 庸言報 [Journal des propos ordinaires] un article intitulé « Minyue pingyi » 《民約平議》 [Critiques du Contrat sociall. ${ }^{2}$ Dans cet article, Yan Fu reprend à son compte les critiques contre Rousseau de Thomas H. Huxley dans On the Natural Inequality of Men, à tel point que Zhang Shizhao 章士釗 publie la même année une critique polémique, signée de son pseudonyme Qiutong 秋桐, dans laquelle il souligne que « rétorquer les arguments à Yan Fu revient à réfuter Huxley lui-même ». ${ }^{3}$ Voyons tout d'abord comment Yan Fu présente les grandes thèses du Contrat social :

商務印書舘 (Editions du Commerce) sous le titre définitif de Shehui qiyuelun 社會契約論. Il est bien rare de voir une œuvre étrangère, en l'occurrence un traité de droit politique écrit pour les spécialistes et non pour le grand public, faire l'objet de si nombreuses traductions et éditions en Chine en l'espace de cent ans. Notre étude n'a pas pour l'objectif de confronter les différentes traductions, ni d'en juger la qualité, mais plutôt de passer au crible les critiques du Contrat social et de tracer le contour de son portrait en Chine.

${ }^{2}$ Yan Fu (1854-1921), penseur chinois, ayant joué un rôle majeur dans l'introduction en Chine de l'évolutionnisme de Darwin, de l'économie capitaliste, de la démocratie libérale anglaise, de la sociologie et de la logique. Entre 1896 et 1912, il traduisit de l'anglais Thomas H. Huxley (Evolution and Ethics), Adam Smith (An Inquiry into the Nature and Cause of the Wealth of Nations), John Stuart Mill (On Liberty), Montesquieu (The Spirit of the Laws), Edward Jenks (A History of Politics).

${ }^{3}$ Zhang Shizhao (1881-1973), écrivain et activiste politique. Il fonda en 1914 Jiayin zazhi 甲寅雜誌 [Revue du tigre], dans laquelle il publia de nombreux articles de réflexion politique dont « Lecture de la Critique du Contrat social de Yan Jidao » datée du 10 mai 1914, voir Zhang Shizhao 章士釗, «Du Yan Jidao 'Minyue pingyi' » 讀嚴幾道 ‘民約平議’ [Lecture de la Critique du Contrat social de Yan Jidao], in Zhang Shizhao quanji 3 章士釗全集 3 [CEuvres complètes de Zhang Shizhao, tome 3], Shanghai, Wenhui chubanshe, 2000, p. 20. Il y déclara qu'il n'était pas un partisan ardent du régime républicain que prônait Rousseau, que la liberté et l'égalité n'étaient pas des privilèges exclusifs d'une république, et que la volonté du peuple pouvait parfois s'exprimer de façon plus vive dans une monarchie constitutionnelle. Quand on met en parallèle l'Angleterre et la Chine, on constate combien cette remarque de Zhang Shizhao reste valable un siècle plus tard. Néanmoins, Zhang Shizhao opposa la république à la 
《(甲)民生而自由者也, 於其擘為平等而皆善, 處於自然, 則常如此。是故自由 平等而樂善者, 其天賦之權利也。（乙）天賦之權利皆同, 無一焉有侵夺其余 之權利。是故公养之物, 莫之能私。如土地及凡土地之所出者, 非人類所同認 公許者不得據之為己有也; 産業者皆篡而得之者也。（丙）箨之權利, 以公約 為之基; 戰勝之權利, 非權利也。凡物之以力為有者, 義得以力而夺之。 4 »

«Primo, les hommes sont nés libres, égaux et bons, tel est l'état de nature. Par conséquent, la liberté, l'égalité et l'amour de la bonté sont les droits naturels de l'homme. Secundo, les droits naturels étant identiques, nul n'a le droit d'usurper sur celui des autres. Nul ne peut s'approprier les biens publics. Par exemple, la terre, de même que sa production ne peut faire l'objet de l'appropriation privée sans le consentement de tous; les propriétaires sont tous des usurpateurs. Tertio, les droits du peuple sont fondés sur le pacte social; la guerre ne donne point le droit au vainqueur. Tout ce que l'on obtient par la force peut aussi être ravi par la force. »

Pour l'auteur du Contrat social, la liberté est un droit inaliénable, parce que c'est un droit naturel. On se souvient du fameux incipit du Contrat social : «L'homme est né libre, et partout il est dans les fers ». Mais selon Yan Fu et son mentor anglais, la liberté et l'égalité ne relèvent nullement de la nature humaine. Ils accusent Rousseau d'avoir supposé a priori le principe du droit naturel en imaginant un «état de nature » qui n'a jamais existé dans l'histoire. Une expérience politique qui n'est pas fondée sur les faits historiques ne relève à leurs yeux que de la pure utopie et ne pourrait que porter préjudice à l'humanité. ${ }^{5}$

Le raisonnement du Contrat social est en fait de s'opposer à l'histoire au nom du droit. Sur ce point, les commentateurs de Rousseau divergent dès le départ. Dans le Manuscrit de Genève, Rousseau précise l'objectif de sa démarche: " Je cherche le droit et la raison et ne discute pas des faits. ». ${ }^{6}$ Il désigne par « l'état de nature » la situation de l'homme avant l'entrée en société. C'est dans cette optique que Rousseau porte un jugement très sévère sur la méthode du juriste hollandais Grotius: «Sa plus constante manière de raisonner est d'établir toujours le droit par le fait. On pourrait employer une méthode plus conséquente, mais non pas plus

monarchie alors que dans la pensée de Rousseau la république ne désigne pas un type particulier de gouvernement, mais le corps politique légitime : «J'appelle donc République tout Etat régi par des lois, sous quelque forme d'administration que puisse être ... Tout gouvernement légitime est républicain. ». Dans la note qui suit, il ajoute : «Pour être légitime il ne faut pas que le gouvernement se confonde avec le souverain, mais qu'il en soit le ministre : alors la monarchie elle-même est république. ». Voir JeanJacques Rousseau, Du Contrat social, Collection « les Intégrales de philo », Paris, Nathan, p. 58.

${ }^{4} \mathrm{Yan} \mathrm{Fu}$ 嚴復, “'Minyue' pingyi » ‘民約'平議 [Critiques du Contrat social], in Zhongguo xiandai xueshu jingdian - Yan Fu juan 中國現代學術經典 - 嚴復卷 [Livres canoniques de la Chine moderne - Yan Fu], Shijiazhuang, Hebei jiaoyu chubanshe, 1996, p. 609.

${ }^{5}$ Yan Fu, «'Minyue' pingyi », p. 611.

6Jean-Jacques Rousseau, CEuvres complètes, tome III, La Pléiade, Gallimard, p. 297. 
favorable aux tyrans. $»^{7}$ La méthode de Rousseau consiste à montrer, au contraire de celle de Grotius, que le droit, étant le devoir-être, ne peut pas être déterminé et fondé à partir de la réalité factuelle, car ce qui existe n'est pas nécessairement légitime. Liang Qichao 梁啓超, en qualifiant de frivolité les attaques de ce genre contre $D u$ Contrat social, affirme dans son article "Lusuo xue'an» 盧梭學案 [L'affaire Rousseau] que c'est Kant qui saisit au mieux le vrai sens du livre de Rousseau :

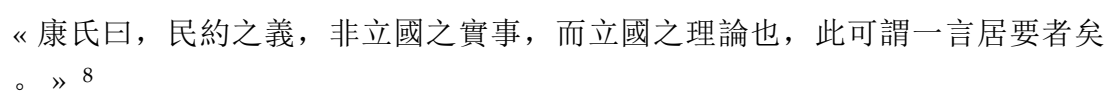

« Kant dit que le contrat social n'a pas à être considéré comme un fait, mais comme une idée du fondement de l'Etat. D'un seul mot, il saisit la clef du problème. »

Dans son essai sur « Kant et Rousseau », Ernst Cassirer tient des propos similaires :

«Kant savait au contraire saisir sans ambiguïté l'essentiel de sa pensée et savait lui donner une formulation claire et sans équivoque. Comme dans sa critique de la connaissance, il distingue très rigoureusement dans sa philosophie du droit la question du quid juris et celle du quid facti. Non seulement la question de l'existence historique du contrat social n'a pas d'importance à ses yeux, mais il considère même que ce contrat ne saurait exister comme un fait, ce qui, affirme-t-il, ne lui enlève en rien sa signification, ou son caractère de certitude. $»^{9}$

Liang Qichao et Ernst Cassirer veulent démontrer que le contrat social, comme "une simple idée de la raison ", consiste à établir le fondement légitime du droit, et non pas à faire état d'une réalité historique. De ce fait, Du Contrat social ne peut se

7Rousseau, Du Contrat social, pp. 32-33.

${ }^{8}$ Liang Qichao 梁啓超, «Lusuo xuan'an» 盧梭學案 [L'affaire Rousseau], in Yinbingshi heji 飲冰室合集 [Recueil des écrits réunis de Liang Qichao], Beijing, Zhonghua shuju, 1988, p. 99. Liang Qichao (18731929), lettré réformiste, écrivain et homme politique. Disciple de Kang Youwei, il dut s'enfuir au Japon après l'échec de la Réforme des Cent Jours (1898). Il y fonda le Bao Huanghui 保皇會 (Association pour la Protection de l'Empereur), et continua à défendre l'idée d'une monarchie constitutionnelle. Les propos de Kant sont beaucoup plus élaborés que le résumé laconique de Liang Qichao. Ernst Cassirer les cite dans son essai sur « Kant et Rousseau »: Un tel contrat « n'a absolument pas à être nécessairement présupposé comme fait (il est d'ailleurs absolument impossible de le présupposer comme fait) », il ajoute que « c'est une simple idée de la raison qui possède néanmoins sa réalité (pratique) indubitable: qui consiste à obliger toute personne qui légifère à produire ses lois de telle façon qu'elles puissent être nées de la volonté unie de tout un peuple et à considérer tout sujet, dans la mesure où il veut être citoyen, comme ayant donné son suffrage à une telle volonté. Car c'est la pierre de touche de la conformité au droit de toute loi publique. », voir Ernst Cassirer [traduit et présenté par Jean Lacoste], Rousseau, Kant, Goethe : deux essais, Belin, 1991, p. 65.

${ }^{9}$ Cassirer, Rousseau, Kant, Goethe, deux essais, p. 65. 
référer qu'à une pure hypothèse normative permettant d'analyser et juger l'état actuel. comme Rousseau écrit déjà dans l'Émile: « Le contrat social est donc la base de toute société civile, et c'est dans la nature de cet acte qu'il faut chercher celle de la société qu'il forme. $»^{10}$

La deuxième critique de $\mathrm{Yan} F \mathrm{Fu}$ porte sur l'appropriation des biens publics dénoncée par Rousseau dans le Discours sur l'origine et les fondements de l'inégalité parmi les hommes. ${ }^{11}$ Dans $D u$ Contrat social, Rousseau relativise son cri de révolte par une déclaration plus nuancée : "Tout homme a naturellement droit à tout ce qui lui est nécessaire $»{ }^{12}$ En comparant le droit de propriété avec le droit de souveraineté dans l'Émile, Rousseau affirme même que :

«si c'est sur le droit de propriété qu'est fondée l'autorité souveraine, ce droit est celui qu'elle doit le plus respecter ; il est inviolable et sacré pour elle tant $\mathrm{qu}^{\prime}$ il demeure un droit particulier et individuel $\ldots »^{13}$

En revenant au droit du premier occupant, Rousseau pose trois conditions:

«Premièrement que ce terrain ne soit encore habité par personne ; secondement qu'on n'en occupe que la quantité dont on a besoin pour subsister ; en troisième lieu qu'on en prenne possession, non par une vaine cérémonie, mais par le travail et la culture, seul signe de propriété qui au défaut de titres juridiques doive être respecté $\mathrm{d}^{\prime}$ autrui. ${ }^{14}$

L'idée que le droit du premier occupant doit être complété par le travail de la terre est reprise par Rousseau dans l'Émile, ce dernier prenant possession de sa terre «en y plantant une fève »: "Et lui expliquant alors ce terme d'appartenir, je lui fais sentir qu'il a mis là son temps, son travail, sa peine, sa personne enfin. $»^{15}$ En prenant le mot «usurpation» au sens étymologique du terme qui veut dire "appropriation par usage», nous pouvons effectivement admettre que «les propriétaires sont tous des usurpateurs ", mais la démarche de Rousseau consiste à changer l'usurpation en un véritable droit, et la jouissance en propriété, même si au fond de lui-même, il restait convaincu que l'usurpation et la propriété sont à

10Jean-Jacques Rousseau, Émile ou de l'éducation, Paris, Garnier, 1964, p. 587.

$11_{«}$ Le premier qui, ayant enclos un terrain, s'avisa de dire: Ceci est à moi, et trouva des gens assez simples pour le croire, fut le vrai fondateur de la société civile. Que de crimes, de guerres, de meurtres, que de misères et d'horreurs n'eût point épargnés au genre humain celui qui, arrachant les pieux ou comblant le fossé, eût crié à ses semblables: Gardez-vous d'écouter cet imposteur ; vous êtes perdus, si vous oubliez que les fruits sont à tous, et que la terre n'est à personne. ", Jean-Jacques Rousseau, Discours sur l'origine de l'inégalité parmi les hommes, Collection « les Intégrales de philo », Paris, Nathan, p. 82.

${ }^{12}$ Rousseau, Du Contrat social, p. 45.

${ }^{13}$ Rousseau, Émile ou de l'éducation, p. 589.

${ }^{14}$ Rousseau, Du Contrat social, p. 45.

${ }^{15}$ Rousseau, Émile ou de l'éducation, p. 120 
l'origine de l'inégalité sociale. Ainsi il se prononce en faveur d'une propriété limitée

«Dans les faits les lois sont toujours utiles à ceux qui possèdent et nuisibles à ceux qui n'ont rien. D'où il suit que l'état social n'est avantageux aux hommes qu'autant qu'ils ont tous quelque chose et qu'aucun d'eux n'a rien de trop. $»^{16}$

Ce souci égalitaire de Rousseau est sans doute l'une des raisons qui expliquent que les révolutionnaires de tous bords se revendiquent de sa pensée politique, et que $\mathrm{d}$ 'autres condamnent son égalitarisme abstrait. Yan Fu, quant à lui, ne croit pas au régime républicain fondé sur la révolution populaire pour établir l'égalité sociale (sur ce point l'Histoire lui a donné raison). On touche probablement là au fondement de sa pensée anti-rousseauiste: adversaire du constructivisme et partisan de la théorie de l'évolution sociale progressiste de Herbert Spencer et d'Edward Jenks, Yan Fu observe que la Chine est en transition d'une société patriarcale vers la société moderne. Lors de la rencontre avec Sun Yat-sen à Londres en 1905, il s'oppose aux idées républicaines et révolutionnaires de son interlocuteur, en plaidant clairement en faveur d'une réforme par étapes avec comme priorité l'éducation du peuple. Il place son espoir dans les générations qui se renouvelleront progressivement. Mais la réponse de Sun Yat-sen est sans appel:

《竢河之清, 人壽幾何? 君為思想家, 鄙人乃執行家也。»17

"Quel âge aurons-nous quand le fleuve Jaune deviendra limpide? Vous êtes un penseur, moi, je suis un homme d'action. »

Considérant que les critiques de Yan Fu et de Zhang Shizhao s'attardent trop aux détails, et sont trop peu soucieuses des valeurs philosophiques du Contrat social, et que les Chinois de l'époque bien mal instruits de la philosophie politique occidentale ne peuvent saisir la hauteur de vue de Rousseau à travers ces deux critiques, Zhang Xiruo 張奚若 fait paraître en 1920 《Sheyuelun kao » 社約論考 [Etude du contrat social]. ${ }^{18}$ Il s'interroge sur l'origine des théories du contrat politique ou social dans l'histoire de la pensée occidentale et cherche à révéler

\footnotetext{
${ }^{16}$ Rousseau, Du Contrat social, p. 47.

${ }^{17}$ Ouyang Zhesheng 歐陽哲生, «Yan Fu xiansheng xiaozhuan» 嚴復先生小傳 [Brève biographie de Yan $\mathrm{Fu}$ ], in Zhongguo xiandai xueshu jingdian - Yan Fu juan 中國現代學術經典 - 嚴復卷 [Livres canoniques de la Chine moderne - Yan Fu], Shijiazhuang, Hebei jiaoyu chubanshe, 1996, p. 5.

${ }^{18}$ Voir Zhang Xiruo 張奚若, «Sheyuelun kao » 社約論考 [Etude critique du contrat social], in Zhang

Xiruo wenji 張奚若文集 [Recueil des écrits de Zhang Xiruo], Beijing, Qinghua daxue chubanshe, 1989, p. 29. Zhang Xiruo (1889-1973), activiste politique, vétéran de la Révolution de 1911, politologue formé aux Etats-Unis et en Europe entre 1913 et 1924, professeur en Sciences politiques à l'Université de Qinghua (Pékin) à partir de 1929.
} 
l'influence durable de l'idée du contrat comme hypothèse méthodologique dans la vie politique de l'humanité. Après avoir procédé à un inventaire exhaustif d'abord chez les Grecs (Platon, Aristote, Epicure, Lucrèce, Métrodore), ensuite chez les penseurs des $\mathrm{XVI}$, $\mathrm{XVII}^{\mathrm{e}}$ et $\mathrm{XVIII}{ }^{e}$ siècles, tels que Duplessis-Mornay, George Buchanan, Althusius, Richard Hooker, Grotius, Thomas Hobbes et John Locke, et enfin chez les philosophes qui succédèrent à Rousseau, comme Emmanuel Kant et Johann G. Fichte, Zhang Xiruo conclut que les théories du contrat social ne constituent pas un système de pensée cohérent et homogène. ${ }^{19}$ Pourtant, il affirme que quelles que soient les divergences de vision entre ces penseurs, leur principe philosophique reste le même, à savoir que le pouvoir légitime doit être fondé sur le consentement populaire, tel est l'esprit du contrat social qui ne se limite pas à l'institution du gouvernement d'un pays quelconque, mais sert aussi de base au fédéralisme et à la future alliance internationale. ${ }^{20}$

Par ailleurs Zhang Xiruo s'engage dans une autre polémique, celle qui opposait, entre autres, Georg Jellinek et le juriste français Emile Boutmy, sur les origines historiques de la Déclaration des droits de l'homme et du citoyen de 1789. L'Allemand Georg Jellinek a démenti l'influence de Rousseau sur cette déclaration et a démontré que celle-ci n'était qu'un plagiat de la Declaration of Independence et du Bills of Rights des Etats-Unis d'Amérique. ${ }^{21}$ Emile Boutmy a riposté fermement en soutenant que la Déclaration des droits de l'homme et du citoyen donnait la meilleure illustration du Contrat social. ${ }^{22}$ Contrairement à certains polémistes dont la démarche consiste à départager les deux adversaires, Zhang Xiruo n'est pas partisan du dualisme. Pour former une opinion objective et juste sur la question, il la divise en deux: Quelle est la visée de la Déclaration des droits de l'homme et du citoyen? Quelle en est la teneur? Autrement dit, d'où vient l'idée de limiter les pouvoirs de l'Etat par une déclaration des droits de l'individu? Quels sont les

\footnotetext{
${ }^{19}$ Philippe de Duplessis-Mornay (1549-1623), chef calviniste, conseiller de Coligny, puis de Henri IV (avant la conversion de celui-ci au catholicisme), auteur présumé de Vindiciae Contra Tyrannos. George Buchanan (1506-1582), humaniste écossais, précepteur du futur Jacques 1 1 ${ }^{\text {er }} \mathrm{d}^{\prime}$ Angleterre, il prôna une monarchie limitée, auteur de De jure regni apud Scotos. Richard Hooker (1554 ?-1600), philosophe anglais, auteur de Of the Laws of Ecclesiastical Polity. Hugo de Groot, dit Grotius (1583-1645), juriste et diplomate hollandais, auteur du De jure belli ac pacis. Thomas Hobbes (1588-1679), philosophe anglais, auteur du Léviathan. John Locke (1632-1704), philosophe anglais, partisan du libéralisme politique, il considère que la société repose sur un contrat et que le souverain doit obéir aux lois, auteur des deux Traités $d u$ gouvernement. Johann Gottlieb Fichte (1762-1814), philosophe allemand, disciple émancipé de Kant, auteur de Théorie de la science.

${ }^{20}$ Zhang Xiruo, «Sheyuelun kao », p. 58.

${ }^{21}$ Voir Georg Jellinek, Die Erklärung der Menschen - und Bürgerrechte, dritte Auflage, Berlin, 1919, pp. 5-42.

${ }^{22}$ Voir Emile Boutmy, La déclaration des droits de l'homme et du citoyen et M. Jellinek, Annales des Sciences

Politiques, 1902, juillet.
} 
penseurs qui ont inspiré par leurs travaux les articles d'une telle déclaration ?23 Citations à l'appui, Zhang Xiruo démontre que si Rousseau a poursuivi, dans les cinq premiers chapitres du Contrat social, l'idée individualiste brillamment mise en exergue dans le "Discours sur l'origine de l'inégalité parmi les hommes » et «Emile ou de l'éducation", il est devenu, à partir du Chapitre VI jusqu'à la fin du livre, un collectiviste ou un étatiste pur et dur, voire au regard de certains, un absolutiste: ${ }^{24}$

«Ces clauses bien entendues se réduisent toutes à une seule, savoir l'aliénation totale de chaque associé avec tous ses droits à toute la communauté. ${ }^{25}$

$[\ldots]$

De plus, l'aliénation se faisant sans réserve, l'union est aussi parfaite qu'elle ne peut l'être et nul associé n'a plus rien à réclamer. ${ }^{26}$

$[\ldots]$

Chacun de nous met en commun sa personne et toute sa puissance sous la suprême direction de la volonté générale: et nous recevons en corps chaque membre comme partie indivisible du tout. ${ }^{27}$

\section{$[\ldots]$}

Comme la nature donne à chaque homme un pouvoir absolu sur tous ses membres, le pacte social donne au corps politique un pouvoir absolu sur tous les siens, et c'est ce même pouvoir qui, dirigé par la volonté générale, porte, comme j'ai dit, le nom de souveraineté. $»^{28}$

Ici les termes utilisés par Rousseau, tels que «toute la communauté », "l'union », «la suprême direction » et «le corps politique » renvoient tous à l'Etat souverain qui est une personne morale :

«Cette personne publique qui se forme ainsi par l'union de toutes les autres prenait autrefois le nom de Cité, et prend maintenant celui de République ou de corps politique, lequel est appelé par ses membres Etat quand il est passif, Souverain quand il est actif, Puissance en le comparant à ses semblables. $»^{29}$

\footnotetext{
${ }^{23}$ Voir Zhang Xiruo, «Faguo renquan xuanyan de laiyuan wenti » 法國人權宣言的来源問题 [Question des origines de la Déclaration française des droits de l'homme], in Zhang Xiruo wenji 張奚若文集 [Recueil des écrits de Zhang Xiruo], Beijing, Qinghua daxue chubanshe, 1989, pp. 140-141.

${ }^{24} Z$ hang Xiruo, «Faguo renquan xuanyan de laiyuan wenti », p. 143.

${ }^{25}$ Rousseau, $D u$ Contrat social, p. 40.

${ }^{26}$ Rousseau, p. 40.

${ }^{27}$ Rousseau, p. 41.

${ }^{28}$ Rousseau, p. 52.

${ }^{29}$ Rousseau, p. 41.
} 
On se laisse facilement convaincre par le raisonnement de Zhang Xiruo fondé sur les arguments irréfutables que fournit le texte du Contrat social : La Déclaration des droits de l'homme et du citoyen de 1789 avait pour vocation de dresser un mur autour du territoire privé de l'individu et d'en assurer la protection contre d'éventuels abus de pouvoir de l'Etat. Or, la conception de l'Etat de Rousseau, telle qu'elle est exposée dans la théorie de la souveraineté, contraire à l'esprit de cette déclaration, ne pouvait lui donner naissance. Mais si Zhang Xiruo semble partager le point de vue de Georg Jellinek, selon lequel l'idée de limiter les pouvoirs de l'Etat par une déclaration des droits de l'individu venait plutôt des Etats-Unis, il diverge totalement avec Jellinek quand ce dernier affirme que la Déclaration des droits de l'homme et du citoyen est une copie de la déclaration américaine. Il invoque les quelques cinquante mille Cahiers de Doléances destinés aux Etats Généraux ; les Comités de Constitution de l'Assemblée Constituante qui se sont succédés; l'accouchement difficile du texte de la déclaration; les débats houleux entre députés, souvent passionnés, parfois orageux; les délibérations interminables des articles et des amendements; et enfin le caractère inachevé et imparfait de cette déclaration, afin de prouver qu'il est absolument injuste de qualifier de copie américaine ce document historique de première importance. Mais d'où viennent les idées que traduisent les articles de la déclaration? Après avoir examiné minutieusement chaque article, la réponse de Zhang Xiruo est sans équivoque:

«Sur le plan des faits, elle vient des souffrances réelles du peuple français d'alors; sur le plan théorique, elle vient de l'ensemble de la philosophie politique du XVIII e siècle, l'influence américaine est perceptible, mais peu importante. ${ }^{30}$

\section{$[\ldots]$}

Parmi les philosophes politiques du XVIII ${ }^{\mathrm{e}}$ siècle, Locke, Montesquieu, Rousseau sont évidemment les plus importants. ${ }^{31}$

\section{$[\ldots]$}

Bien que la méthode de limiter les pouvoirs de l'Etat au moyen de Déclaration des droits de l'homme ne vienne pas du Contrat social de Rousseau, qu'elle soit même contraire à l'esprit du Contrat social, la pensée et la théorie fondamentales dont sont imprégnés les articles de la Déclaration des droits de l'homme font écho à de la philosophie politique de Rousseau. " ${ }^{32}$

Il en résulte que la Declaration of Independence, la Bills of Rights des Etats-Unis d'Amérique et la Déclaration des droits de l'homme et du citoyen de 1789 sont deux

\footnotetext{
${ }^{30}$ Zhang Xiruo, « Faguo renquan xuanyan de laiyuan wenti », p. 194.

${ }^{31}$ Zhang Xiruo, p. 196.

${ }^{32}$ Zhang Xiruo, p. 198.
} 
filles de la philosophie politique du XVIII siècle dont les droits naturels de l'homme et la souveraineté du peuple constituent deux pierres angulaires.

Certes, la force de l'argumentation de Zhang Xiruo ne peut nous laisser insensibles. Sa démonstration est solide et bien documentée. Il reste peut-être un point qui mérite d'être discuté, c'est la façon dont il définit l'objet de la Déclaration des droits de l'homme et du citoyen, car celle-ci ne sert pas qu'à affirmer «les droits naturels, inaliénables et sacrés de l'homme » et par là à circonscrire les pouvoir de l'Etat, mais aussi à rappeler les devoirs des citoyens. Sachant quels sont ses droits, l'homme sentira qu'il ne peut jouir des siens qu'en respectant ceux des autres et qu'en accomplissant ses devoirs envers l'Etat. C'est aussi en cela que la Déclaration des droits de l'homme et du citoyen rejoint l'esprit du Contrat social.

\section{La critique du Contrat social à la fin du XXe siècle}

En 1986, dix ans après la Révolution culturelle, Chen Weigang 陳維綱 publie dans la revue Dushu 讀書 [Lire] sa lecture du Contrat social. ${ }^{33}$ Il résume l'idée maîtresse de son article par la citation d'un politologue anglais G. R. Gettell:

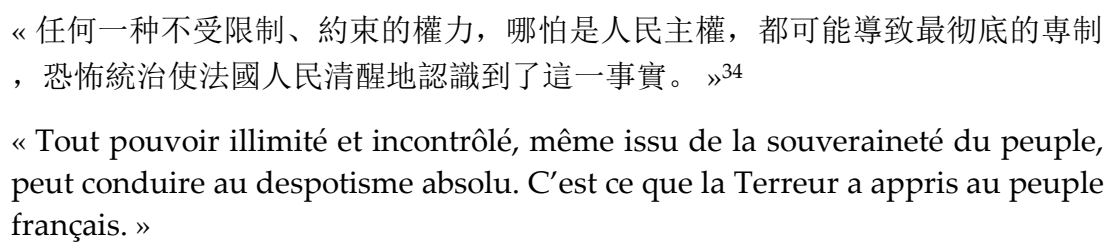

En 1994, Zhu Xueqin 朱學勤 publie sa thèse de doctorat intitulée "La fin de la République de vertu - de Rousseau à Robespierre», travail bien documenté cherchant à établir les liens entre l'auteur du Contrat social et la terreur de la Révolution française, dans le sillage d'Edmund Burke, auteur des Réflexions sur la révolution de France. ${ }^{35}$ En 2001 paraît un ouvrage de Wu Jiaxiang mettant en cause certains concepts clés de Rousseau. ${ }^{36}$ L'auteur considère $\mathrm{Du}$ Contrat social comme

\footnotetext{
${ }^{33}$ Chen Weigang 陳維綱, «Ping Lusuo renmin zhuquanlun de zhuanzhizhuyi qingxiang » 評盧梭人民主 權論的專制主義傾向 [Critique de la tendance au despotisme dans la théorie de la souveraineté du peuple de Rousseau], Dushu, décembre 1986.

${ }^{34}$ Chen Weigang, «Ping Lusuo renmin zhuquanlun de zhuanzhizhuyi qingxiang », p. 32. ${ }^{35}$ Zhu Xueqin 朱學勤, Daode lixiangguo de Fumie - Cong Lusuo dao Luobosibi'er 道德理想國的覆滅——從盧 梭到羅伯斯庇爾 [La fin de la République de vertu, de Rousseau à Robespierre], Shanghai, Shanghai Sanlian shudian, 1994.

${ }^{36} \mathrm{Wu}$ Jiaxiang 吳稼祥, Tou duizhe qiang - Daguo de minzhuhua 頭對著墻一一大國的民主化 [Tête face au mur - La démocratisation du grand pays], Taipei, Lianjing, 2001. Diplômé de la Faculté d'économie de l'Université de Pékin, Wu Jiaxiang a été employé de 1982 au 1989 au bureau de la propagande et au secrétariat général du Parti communiste chinois. Ecarté en 1989 après le massacre de Tiananmen, il a été emprisonné pendant trois ans pour avoir soutenu le mouvement étudiant.
} 
une théorie de pseudo-démocratie, coupable d'avoir fourni un fondement à l'autorité politique du tyran et d'avoir ainsi conféré une légitimité à la tyrannie de tous ceux qui avaient pris le pouvoir par la révolution.

Contrairement à Yan Fu qui conteste au nom de l'histoire empirique l'apriorisme de Rousseau et la vision utopiste du Contrat social, les critiques des intellectuels chinois de la nouvelle génération portent principalement sur les conséquences politiques de cet ouvrage. Wu Jiaxiang affirme que le régime totalitaire communiste correspond parfaitement à la définition rousseauiste de "la souveraineté du peuple ", abstraction faite de la dictature du prolétariat. Reprenant l'idée persistante de Benjamin Constant que «La métaphysique subtile du Contrat social n'est propre, de nos jours, qu'à fournir des armes et des prétextes à tous les genres de tyrannie ${ }^{37}$, Wu Jiaxiang accuse Rousseau d'être à l'origine de la tyrannie contemporaine de I'URSS et de la Chine, tandis que Zhu Xueqin reproche à Rousseau d'avoir tendu sa main vers l'échafaud, alors que ses cendres allaient être transférées au Panthéon :

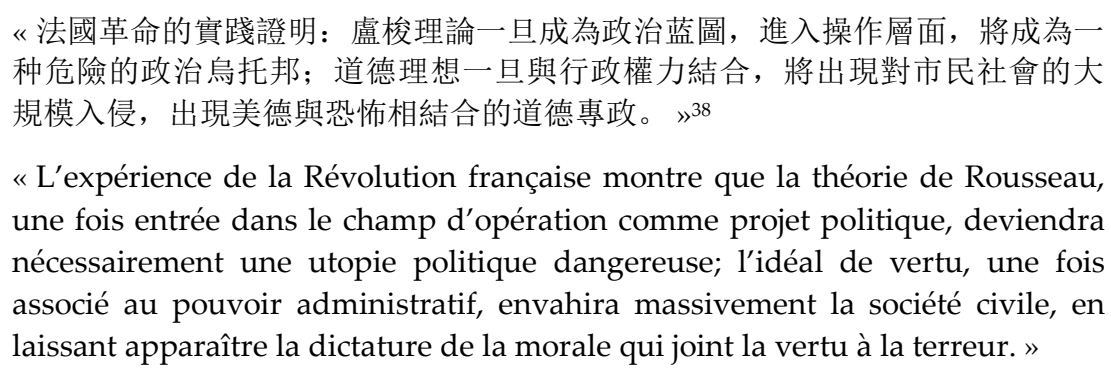

«L'expérience de la Révolution française montre que la théorie de Rousseau, une fois entrée dans le champ d'opération comme projet politique, deviendra nécessairement une utopie politique dangereuse; l'idéal de vertu, une fois associé au pouvoir administratif, envahira massivement la société civile, en laissant apparaître la dictature de la morale qui joint la vertu à la terreur. »

Et la main de Rousseau, c'est bien sûr Robespierre. N'a-t-il pas dit un jour que « la terreur sans vertu est vice, la vertu sans terreur est faiblesse $» .{ }^{39}$ Dix-sept ans après la mort du penseur, son testament spirituel a été mis à exécution à travers le décret du 7 mai $1794 .{ }^{40}$

Un autre concept clé de Rousseau pris comme cible pour son abstraction et son oubli de l'homme concret est «la volonté générale ». A la lumière de ce qui se passe en Chine et ailleurs, Chen Weigang fait observer:

\footnotetext{
37Dans De la liberté des Anciens comparée à celle des Modernes, Folio, Paris, Gallimard, Benjamin Constant cherche à montrer « qu' en transportant dans nos temps modernes une étendue de pouvoir social, de souveraineté collective qui appartenait à d'autres siècles, ce génie sublime qu'animait l'amour le plus pur de la liberté a fourni néanmoins de funestes prétextes à plus d'un genre de tyrannie », p. 604.

${ }^{38}$ Zhu Xueqin, Daode lixiangguo de Fumie - Cong Lusuo dao Luobosibi'er, p. 35.

${ }^{39}$ Zhu Xueqin, p. 256.

${ }^{40} I l$ s'agit du Décret du 18 floréal, l'an II de la République Française (1794), Rapport fait par Maximilien Robespierre à la Convention Nationale, au nom du Comité de Salut Public, sur les rapports des idées religieuses et morales avec les principes républicains, et sur les fêtes nationales.
} 
《人民能够受騙這一事實本身, 就證明了所謂公意不可能永遠正确, 而在一個個 人權利得不到保證的社會中, 就意味著成千上萬的無啈者丧失自由, 丧失生命 。 ${ }^{41}$

«Le fait même que le peuple peut être trompé prouve que la volonté générale ne saurait être toujours juste. Dans une société où les droits de l'individu ne sont point garantis, des dizaines de milliers d'innocents risquent de perdre la liberté et la vie. »

\section{《在他的理想國中, 専制獨裁的危險並非来自政府背叛公意, 而是一個無所不包 的公意本身就意味著専制。»42}

«Dans sa République, le danger de la dictature ne vient pas du gouvernement qui trahit la volonté générale, mais du fait que la volonté générale omnipotente en elle-même est une dictature. »

Wu Jiaxiang va encore plus loin dans son raisonnement déductif. Il constate que dans le vocabulaire de certains révolutionnaires, "la liberté du peuple» est synonyme de "libération». Après la libération, le peuple doit être gouverné en fonction de « la volonté générale ». Hier en Union soviétique, aujourd'hui en Chine, cette "volonté générale » est celle qu'un leader prétendu souverain proclame au peuple tout entier. Qu'est-ce qui peut lui arriver, si quelqu'un ne se soumet pas à " la volonté générale » ? La réponse de Rousseau à cette question répond en même temps aux désirs de tous les tyrans: «...quiconque refusera d'obéir à la volonté générale y sera contraint par tout le corps: ce qui ne signifie autre chose sinon qu'on le forcera d'être libre. ».43 Ce qui paraît le plus absurde et le plus inconcevable à Wu Jiaxiang, c'est que l'auteur du Contrat social va jusqu'à affirmer que «la puissance souveraine n'a nul besoin de garant envers les sujets », ce qui revient à donner carte blanche à celle-là. ${ }^{44}$ Aussi critique-t-il fortement le raisonnement de Rousseau, selon lequel « du silence universel on doit présumer le consentement du peuple ${ }^{45}$ Citant Benjamin Constant pour appuyer son assertion, Wu Jiaxiang juge la tyrannie pire que la dictature aussi bien en brutalité qu'en perversité, car « le souverain », en assimilant le silence à un mécontentement subversif, tend à forcer le peuple à renoncer à sa réserve silencieuse et à se rallier publiquement à « la volonté générale » que « le souverain » est censé représenter. ${ }^{46}$

\footnotetext{
${ }^{41}$ Chen Weigang, « Ping Lusuo renmin zhuquanlun de zhuanzhizhuyi qingxiang », p. 37.

${ }^{42}$ Chen Weigang, p. 40.

${ }^{43}$ Rousseau, Du Contrat social, p. 43.

${ }^{44}$ Rousseau, p. 43.

${ }^{45}$ Rousseau, p. 48.

${ }^{46}$ Il s'agit de la citation suivante: «Le despotisme, en un mot, règne par le silence, et laisse à l'homme le droit de se taire ; l'usurpation le condamne à parler ; elle le poursuit dans le sanctuaire intime de sa pensée, et, le forçant à mentir à sa conscience, elle lui ravit la dernière consolation qui reste encore à
} 
Pour saisir le principe de la «souveraineté du peuple» dans sa totalité, il faut préciser qu'à son époque, Rousseau voulait dénoncer l'Europe monarchique, aristocratique et cléricale. La préoccupation historique du moment était de transformer le monopole du pouvoir politique détenu par la royauté en souveraineté populaire, d'où les attaques lancées par Rousseau, d'une part contre Grotius qui justifiait la monarchie absolue et le droit de conquête, d'autre part contre Montesquieu, parce que «l'immortel auteur de l'Esprit des lois s'est montré le partisan zélé des inégalités et des privilèges ».47 Benjamin Constant, en débit de sa répugnance contenue pour la pensée politique de Rousseau, partageait l'aversion de celui-ci pour l'Ancien Régime comme étant " un mélange de corruption, d'arbitraire et de faiblesse». Il rappelait que Rousseau "a déclaré que la souveraineté ne pouvait être ni aliénée, ni déléguée, ni représentée. C'était déclarer en d'autres termes qu'elle ne pouvait être exercée ; c'était anéantir de fait le principe $q^{\prime}$ 'il venait de proclamer.»48 On peut relever d'autres contradictions de ce genre dans $D u$ Contrat social: par exemple, dans le livre premier, chapitre VI, Rousseau a défini la nature du pacte social en mettant l'accent sur l'aspect autonome et volontaire de l'association:

«Trouver une forme d'association qui défende et protège de toute la force commune la personne et les biens de chaque associé, et par laquelle chacun s'unissant à tous n'obéisse pourtant qu'à lui-même et reste aussi libre qu'auparavant. Tel est le problème fondamental dont le contrat social donne la solution. » 49

Mais dans le chapitre VII, Rousseau semblait insister sur le côté hétéronome et imposé du contrat social :

"Afin donc que le pacte social ne soit pas un vain formulaire, il renferme tacitement cet engagement qui seul peut donner de la force aux autres, que quiconque refusera d'obéir à la volonté générale y sera contraint par tout le corps: ce qui ne signifie autre chose sinon qu'on le forcera d'être libre. » ${ }^{50}$

Il est clair que Rousseau tenait alternativement deux langages antagonistes, celui du contrat ou du consentement et celui de l'adhésion forcée à la société. Cette vision ambivalente échappe complètement aux analyses de $\mathrm{Wu}$ Jiaxiang qui, en

l'opprimé. », Benjamin Constant, « De l'esprit de conquête et de l'usurpation », in Ecrits politiques, Folio, Paris, Gallimard, p. 197.

${ }^{47}$ Hugo Grotius, Le droit de la guerre et de la paix, Paris, PUF, 2005.

${ }^{48}$ Constant, «Principes de politique », in Écrits politiques, p. 314.

${ }^{49}$ Rousseau, Du Contrat social, p. 40.

${ }^{50}$ Rousseau, Du Contrat social, p. 43. 
reprochant à Rousseau d'avoir prôné « la liberté du peuple» au détriment de «la liberté de l'individu », conclut de façon catégorique:

\title{
《以洛克為代表的人權本位思想奠定了當代自由民主制度的基礎, 而以盧梭為代 表的[人民主權]也就是[人民集權]理論，是當代僭主政治的思想来源。”
}

\begin{abstract}
«La pensée centrée sur les droits de l'homme que représente Locke a jeté les bases du régime de la démocratie libérale, alors que la théorie de «la souveraineté du peuple», c'est-à-dire «la concentration du pouvoir du peuple », est à l'origine de la pensée inspirant la tyrannie contemporaine. »
\end{abstract}

Il est sans doute plus pertinent d'évoquer la double définition de l'émancipation dans le monde moderne, qui donne lieu aux deux traditions démocratiques : l'une libérale, l'autre radicale; l'une individualiste, l'autre collective; l'une dérivée de Locke, l'autre de Rousseau. Pierre Rosanvallon décrit cette double définition de façon distincte:

«La définition de l'émancipation-autonomie est donnée par Locke. Le but de l'émancipation est dans ce cadre de construire l'individu toujours plus indépendant des autres, alors que la vision rousseauiste de l'émancipation est de rendre chacun plus fort à travers sa participation à la collectivité. L'histoire de la modernité s'est déployée sous la forme d'une concurrence et d'un conflit permanent entre ces deux approches. La tension entre l'émancipationautonomie et l'émancipation-puissance renvoie là tout simplement à l'écart du libéralisme à la démocratie (l'un représentant le moment de l'autonomie, et l'autre exprimant le moment de la puissance collective). $\gg^{52}$

On peut interpréter cette distinction que fait Rosanvallon à la lumière de la Révolution française, notamment de la Terreur. Comme l'émancipation-puissance peut entrer en conflit avec l'émancipation-autonomie, il faut imposer certaines limites à « la souveraineté du peuple » pour que celle-ci ne dépasse pas sa propre sphère en faisant irruption sur le territoire de l'individu. "L'écart du libéralisme à la démocratie » dont parle Rosanvallon est ainsi réduit par le régime de la démocratie libérale qui est une synthèse ou un compromis des exigences de Locke et de Rousseau. Quelle que soit la faiblesse du raisonnement de Rousseau, on ne peut mettre en cause son postulat de la légitimité du pouvoir. Ce n'est pas parce qu'une idée noble et juste a été l'objet d'horribles abus que son principe premier doit être renié.

Dans son livre, Wu Jiaxiang souscrit à l'idée courante que Rousseau est hostile à toute séparation des pouvoirs. Mais quand on examine de près la position de

${ }^{51} \mathrm{Wu}$ Jiaxiang 吳稼祥, Tou duizhe qiang - Daguo de minzhuhua, pp. 143-144.

52Pierre Rosanvallon, « Le déficit démocratique européen », Esprit, octobre 2002, pp. 91-92. 
Rousseau sur cette question, elle ne paraît pas aussi tranchée. Dans le livre II, chapitre II du Contrat social, il s'en prenait effectivement aux politiques et les comparait aux charlatans du Japon:

«Mais nos politiques ne pouvant diviser la souveraineté dans son principe la divisent dans son objet; ils la divisent en force et en volonté, en puissance législative et en puissance exécutive, en droits d'impôts, de justice, et de guerre, en administration intérieure et en pouvoir de traiter avec l'étranger: tantôt ils confondent toutes ces parties et tantôt ils les séparent; ils font du souverain un être fantastique et formé de pièces rapportées ; c'est comme s'ils composaient l'homme de plusieurs corps dont l'un aurait des yeux, l'autre des bras, l'autre des pieds, et rien de plus. Les charlatans du Japon qui dépècent, dit-on, un enfant aux yeux des spectateurs, puis jetant en l'air tous ses membres l'un après l'autre, ils font retomber l'enfant vivant en tout rassemblé. „53

Mais dans le livre III, chapitre XVI, il semblait opter pour la séparation naturelle du pouvoir législatif et du pouvoir exécutif:

«Le pouvoir législatif une fois bien établi, il s'agit d'établir de même le pouvoir exécutif ; car ce dernier, qui n'opère que par des actes particuliers, n'étant pas de l'essence de l'autre, en est naturellement séparé. S'il était possible que le souverain, considéré comme tel, eût la puissance exécutive, le droit et le fait seraient tellement confondus qu' on ne saurait plus ce qui est loi et ce qui ne l'est pas, et le corps politique ainsi dénaturé serait bientôt en proie à la violence contre laquelle il fut institué. „\$4

Rousseau était conscient de la contradiction à laquelle il a exposé ses idées sur la séparation ou la non séparation des pouvoirs. Dans le livre III, chapitre IV traitant le problème de la démocratie, il s'expliquait en ces termes:

«Celui qui fait la loi sait mieux que personne comment elle doit être exécutée et interprétée. Il semble donc qu'on ne saurait avoir une meilleure constitution que celle où le pouvoir exécutif est joint au législatif. Mais c'est cela même qui rend ce gouvernement insuffisant à certains égards, parce que les choses qui doivent être distinguées ne le sont pas, et que le prince et le souverain n'étant que la même personne, ne forment, pour ainsi dire, qu'un gouvernement sans gouvernement. Il n'est pas bon que celui qui fait les lois les exécute, ni que le corps du peuple détourne son attention des vues générales, pour la donner aux objets particuliers. „ ${ }^{55}$

${ }^{53}$ Rousseau, Du Contrat social, p. 49.

${ }^{54}$ Rousseau, p. 108.

${ }^{55}$ Rousseau, Du Contrat social, pp. 81-82. 
Il est clair que Rousseau tenait une fois encore deux langages contradictoires, mais il est difficile de dire après cet examen qu'il fut totalement hostile à la séparation des pouvoirs.

L'un des concepts clés du Contrat social est celui de la loi. Pour Rousseau, la première vertu de la loi, c'est qu'elle permet aux hommes d'être libres et de se sortir de la servitude, comme il sut si bien l'expliquer dans les Lettres écrites de la montagne:

«Un peuple libre obéit, mais il ne sert pas ; il a des chefs, et non des maîtres ; il obéit aux Loix, mais il n'obéit qu'aux Loix ; et c'est par la force des Loix qu'il n'obéit pas aux hommes. ${ }^{56}$

Ce précepte de la raison est sans doute partagé par les Lumières. L'article «Économie politique » de l'Encyclopédie a fait de la loi une condition déterminante de la liberté et de l'égalité:

"C'est à la loi seule que les hommes doivent la justice et la liberté, c'est cet organe salutaire de la volonté de tous qui rétablit dans le droit l'égalité naturelle entre les hommes. $\gg^{57}$

Benjamin Constant, malgré les critiques sévères qu'il adressait à Rousseau, a reconnu que parmi les grands publicistes du XVIII siècle, Rousseau avait, "le premier, rendu populaire le sentiment de nos droits ».58 Ernst Cassirer a commenté l'enthousiasme de Rousseau pour la loi en ces termes:

«Par conséquent, pour Rousseau, le lien social véritable vient du fait qu'aucun individu, aucun groupe particulier n'est appelé à gouverner les autres, car un tel gouvernement, quelque raffinée ou civilisée qu'en fût la forme, ne pourrait que nous réduire au plus abject des esclavages. Cet esclavage ne disparaît que si la loi, en tant que telle, guide et dirige les hommes, et que si l'individu, dans son commerce avec les autres hommes, est libre de toute sujétion envers autrui, une commune obéissance à la loi se substituant aux liens d'allégeance et d'obéissance. ${ }^{59}$

Une loi peut être révoquée par la volonté générale, car « un peuple est toujours le maître de changer ses lois, même les meilleures ", mais un individu ne peut pas

\footnotetext{
56Jean-Jacques Rousseau, Lettres écrites de la montagne, in CEuvres complètes, tome III, La Pléiade, Gallimard, 1985, p. 842.

${ }^{57}$ Rousseau, Du Contrat social, p. 151.

58Stephen Holmes, Benjamin Constant et la genèse du libéralisme moderne, collection « Léviathan », PUF, 1994, p. 122.

${ }^{59}$ Cassirer, Rousseau, Kant, Goethe, deux essais, pp. 60-61.
} 
décider qu'une loi n'est plus valable et lui désobéir.60 Pour que le système juridique garantisse l'égalité des citoyens, il faut que la loi s'applique à tous, sans distinction de fortune ou de statut social. On peut aussi interpréter Rousseau dans ce sens, lorsqu'il écrivait:

«...quiconque refusera d'obéir à la volonté générale y sera contraint par tout le corps: ce qui ne signifie autre chose sinon qu'on le forcera d'être libre; car telle est la condition qui donnant chaque citoyen à la Patrie le garantit de toute dépendance personnelle; condition qui fait l'artifice et le jeu de la machine politique, et qui seule rend légitimes les engagements civils, lesquels sans cela seraient absurdes, tyranniques, et sujets aux plus énormes abus. »61

L'interprétation d'une idée équivoque dépend souvent du point de vue de l'interprète. Si l'on se met du côté du peuple, le paragraphe en question offre plutôt une garantie de ne pas tomber dans la dépendance d'un individu qui se met audessus des lois.

Deux autres concepts clés qui occupent une place centrale dans le Contrat social sont ceux de la «souveraineté » et du «gouvernement». Rousseau a distingué le souverain, auteur des lois et le gouvernement chargé de leur exécution. Il a d'abord défini le souverain comme une personne morale:

«Cette personne publique qui se forme ainsi par l'union de toutes les autres prenait autrefois le nom de Cité, et prend maintenant celui de République ou de corps politique, lequel est appelé par ses membres État quand il est passif, Souverain quand il est actif, Puissance en le comparant à ses semblables. A l'égard des associés, ils prennent collectivement le nom du Peuple, et s'appellent en particulier citoyens comme participant à l'autorité souveraine, et sujets comme soumis aux lois de l'État. »62

Quant au gouvernement, Rousseau l'a défini comme un corps de magistrats subordonné au souverain dont il est le serviteur. Autrement dit, le gouvernement n'est pas une partie de la souveraineté:

«Qu'est-ce donc que le gouvernement ? Un corps intermédiaire établi entre les sujets et le souverain pour leur mutuelle correspondance, chargé de l'exécution des lois et du maintien de la liberté, tant civile que politique. »63

\footnotetext{
${ }^{60}$ Rousseau, Du Contrat social, p. 72.

${ }^{61}$ Rousseau, p. 43.

${ }^{62}$ Rousseau, p. 41.

${ }^{63}$ Rousseau, Du Contrat social, p. 74.
} 
Si le souverain détient la puissance législative, le gouvernement détient la puissance exécutive au service de la volonté libre du souverain qui peut le révoquer à sa guise:

"J'appelle donc gouvernement ou suprême administration l'exercice légitime de la puissance exécutive, et prince ou magistrat l'homme ou le corps chargé de cette administration. ${ }^{64}$

\section{$[\cdots]$}

...les dépositaires de la puissance exécutive ne sont point les maitres du peuple mais ses officiers, qu'il peut les établir et les destituer quand il lui plaît, qu'il n'est point question pour eux de contracter mais d'obéir, et qu'en se chargeant des fonctions que l'État leur impose, ils ne font que remplir leur devoir de citoyens, sans avoir en aucune sorte le droit de disputer sur les conditions. $»^{65}$

Dans une note du Livre II, Chapitre VI, Rousseau a même fait de cette distinction le critère du jugement de la légitimité du gouvernement:

«Pour être légitime il ne faut pas que le gouvernement se confonde avec le souverain, mais qu'il en soit le ministre: alors la monarchie elle-même est république. ${ }^{66}$

Pour répondre à l'irritation de $\mathrm{Wu}$ Jiaxiang contre les propos de Rousseau, tels que «du silence universel on doit présumer le consentement du peuple», il faudrait sans doute se rapporter au contexte, car de tels propos peuvent paraitre justifiés dans leur contexte, mais inadmissible dans un autre:

«Ce n'est point à dire que les ordres des chefs ne puissent passer pour des volontés générales, tant que le souverain libre de s'y opposer ne le fait pas. En pareil cas, $\mathrm{du}$ silence universel on doit présumer le consentement du peuple. » ${ }^{67}$

Afin de présumer du silence universel le consentement du peuple, une condition s'impose: «tant que le souverain libre de s'y opposer ne le fait pas». Dans le contexte de Rousseau, le souverain est un peuple libre. Il a non seulement le droit de s'opposer aux ordres des chefs, et il peut effectivement le faire. Ce droit de contredire et cette liberté d'opposition supposent un pays démocratique dont le peuple est souverain. Or la situation dont parle $\mathrm{Wu}$ Jiaxiang est tout autre. Il s'agit d'un usurpateur déguisé en souverain qui, par la répression, force le peuple à se

${ }^{64}$ Rousseau, p. 74.

${ }^{65}$ Rousseau, pp. 110-111.

${ }^{66}$ Rousseau, p. 58.

${ }^{67}$ Rousseau, p. 48. 
taire et à se soumettre à sa volonté particulière. Dans ce cas, si « le peuple promet simplement d'obéir, il se dissout par cet acte, il perd sa qualité de peuple; à l'instant qu'il y a un maître il n'y a plus de souverain.».68 Benjamin Constant cité par Wu Jiaxiang, parlait lui aussi de l'usurpation comparée au despotisme. Il ne s'agit pas non plus d'un peuple libre. Il semble bien que Rousseau tient le gouvernement en suspicion, car quelle que soit sa forme, il a toujours tendance à dégénérer, en substituant sa propre volonté à la volonté générale. Tout l'art de la politique consiste à lutter contre les empiétements du gouvernement sur le souverain.

« S'il arrivait enfin que le prince eût une volonté particulière plus active que celle du souverain, et qu'il usât, pour obéir à cette volonté particulière, de la force publique qui est dans ses mains, en sorte qu'on eût, pour ainsi dire, deux souverains, l'un de droit, l'autre de fait; à l'instant l'union sociale s'évanouirait, et le corps politique serait dissout. ${ }^{69}$

\section{$[\ldots]$}

Car en paraissant n'user que de ses droits il lui est fort aisé de les étendre, et d'empêcher sous le prétexte du repos public, les assemblées destinées à rétablir le bon ordre ; de sorte qu'il se prévaut d'un silence qu'il empêche de rompre, ou des irrégularités qu'il faut commettre, pour supposer en sa faveur l'aveu de ceux que la crainte fait taire, et pour punir ceux qui osent parler. » ${ }^{70}$

Il convient donc de s'offrir le moyen de prévenir les usurpations du gouvernement. $C^{\prime}$ est la raison pour laquelle Rousseau insistait pour que la tenue des assemblées du peuple se fassent «toujours par deux propositions qu'on ne puisse jamais supprimer, et qui passent séparément par les suffrages. La première : S'il plaît au souverain de conserver la présente forme de gouvernement. La seconde : S'il plaît au peuple d'en laisser l'administration à ceux qui en sont actuellement chargés $» .^{71}$ Ce rôle que Rousseau attribuait à l'ouverture des assemblées du peuple est celui des élections présidentielles ou législatives dans les démocraties d'aujourd'hui. Les modes de légitimation et de surveillance peuvent varier, mais la souveraineté démocratique s'exprime toujours par la procédure électorale du suffrage.

En prenant quelques risques de simplification et en guise de conclusion temporaire, nous relèverons trois points importants concernant la critique de la critique chinoise du Contrat social:

\footnotetext{
${ }^{68}$ Rousseau, Du Contrat social, p. 48.

${ }^{69}$ Rousseau, p. 77.

${ }^{70}$ Rousseau, p. 111.

${ }^{71}$ Rousseau, p. 112.
} 
1. En dépit du culte extraordinaire rendu à Rousseau par les acteurs de la Révolution française, il serait prudent de relativiser l'influence de Rousseau sur la Révolution elle-même. Celle-ci a mis en œuvre un système représentatif que Rousseau rejetait en s'opposant clairement à ce que le peuple puisse déléguer le pouvoir législatif à des représentants. Or c'est justement ce système représentatif qui a rendu possible la dictature illimitée des Jacobins, responsable de la Terreur. De ce fait, on ne peut prétendre que la Révolution française est purement et simplement rousseauiste. Dans sa critique de Rousseau, $\mathrm{Wu}$ Jiaxiang reproche à celui-ci, d'une part de "s'opposer aux groupes d'intérêt de tout genre », et d'autre part de faire de la théorie de "souveraineté du peuple" la source même de la tyrannie contemporaine..$^{72}$ Or loin d'avoir imposé une sorte de dictature de la volonté générale, le totalitarisme du XXe siècle a instauré en URSS comme en Chine la dictature d'un parti unique, alors que, comme $\mathrm{Wu}$ Jiaxiang le rappelle, Rousseau rejetait le principe même des partis politiques. La République de Rousseau n'est ni un «État de prince», ni un "État-parti», elle est un État régi par des lois: «J'appelle donc République tout État régi par des lois, sous quelque forme d'administration que ce puisse être... » Le régime totalitaire du parti unique est par définition contraire à la conception rousseauiste de «la souveraineté du peuple » et de la République. ${ }^{73}$

2. Rousseau a décrit dans le Contrat social l'État corrompu où la souveraineté en tant que l'autorité suprême de l'État n'est plus l'exercice de la volonté générale :
«Enfin quand l'État près de sa ruine ne subsiste plus que par une forme illusoire et vaine, que le lien social est rompu dans tous les cœurs, que le plus vil intérêt se pare effrontément du nom sacré du bien public, alors la volonté générale devient muette; tous guidés par des motifs secrets n'opinent pas plus comme citoyens que si l'État n'eût jamais existé, et l'on fait passer faussement sous le nom de lois des décrets iniques qui n'ont pour but que l'intérêt particulier. $\gg^{74}$

Benjamin Constant, plusieurs fois cité par Wu Jiaxiang comme détracteur de Rousseau ne voyait pas le péril «dans l'existence du gouvernement du peuple par lui-même, mais plutôt dans l'apparition éventuelle de dictatures habiles capables de manipuler un régime qui n'aurait plus que le nom de démocratie $» .{ }^{75} \mathrm{Si}$ le régime issu de la Révolution française était bien démocratique, en ce qu'il procédait

\footnotetext{
${ }^{72} \mathrm{Wu}$ Jiaxiang, Tou duizhe qiang - daguo de minzhuhua, p. 138, p. 144.

${ }^{73}$ Rousseau, Du Contrat social, p. 58.

${ }^{74}$ Rousseau, pp. 113-114.

75Holmes, Benjamin Constant et la genèse du libéralisme moderne, p. 135.
} 
de la souveraineté du peuple, le despotisme des Jacobins, qui se nommaient encore les représentants du peuple, n'était pas «la tyrannie de la majorité, mais l'accaparement du pouvoir par quelques-uns $» .{ }^{76}$

3. Il faudrait enfin ne pas oublier ni même négliger le fait que la pensée de Rousseau se présente avant tout comme une norme pour critiquer les sociétés établies. Ceci explique pourquoi aucun des systèmes démocratiques existants n'a mis en œuvre complètement la conception rousseauiste de la souveraineté du peuple en tant qu'exercice de la volonté générale, et qu'ils n'en laissent voir tout au plus que quelques formes embryonnaires. L'objectif de son ouvrage, tel qu'il l'a fixé dans la brève conclusion du Contrat social, est de poser les vrais principes du droit politique et de tâcher de fonder l'Etat sur cette base. Dans son magistral ouvrage, Théorie de la justice, John Rawls s'emploie à élucider la démarche contractualiste de Rousseau dont il se place lui-même comme l'héritier:

«Mon but est de présenter une conception de la justice qui généralise et porte à un plus haut niveau d'abstraction la théorie bien connue du contrat social telle qu'on la trouve, entre autres, chez Locke, Rousseau et Kant. Pour cela, nous ne devons pas penser que le contrat originel soit conçu pour nous engager à entrer dans une société particulière ou pour établir une forme particulière de gouvernement. L'idée qui nous guidera est plutôt que les principes de la justice valables pour la structure de base de la société sont l'objet de l'accord originel. $»^{77}$

Zhu Xueqin, en dépit de sa critique sévère contre l'idéal de vertu proposé par Rousseau reconnaît que la pensée de celui-ci pourrait connaître une deuxième floraison auprès des sociétés industrielles et post-industrielles, non pas comme programme politique à caractère utopique, mais en tant qu'un héritage de valeurs pour le mouvement des critiques sociales. ${ }^{78}$ De la division des Lumières et de l'échec de la Révolution française, Hegel aurait tiré sa fameuse conclusion paradoxale: «Tout ce qui existe est juste; tout ce qui est juste existe ». La première phrase résume l'empirisme de Locke et de Voltaire ; la deuxième, l'apriorisme de Descartes et de Rousseau. La maxime de Hegel se confirme à travers l'histoire

\footnotetext{
${ }^{76}$ Holmes, p. 135.

77John Rawls, Théorie de la justice sociale, Paris, Seuil, 1997, p. 37. Dans une note sur ce passage, John Rawls précise: «Comme le suggère le texte, je considérerai comme étant des œuvres décisives dans la théorie du contrat The Second Treatise of Government de Locke, Du contrat social de Rousseau, et l'éthique de Kant qui débute par Les Fondements de la métaphysique des mœurs. », p. 79.

${ }^{78}$ Zhu Xueqin, Daode lixiangguo de Fumie - Cong Lusuo dao Luobosibi'er, p. 280.
} 
bicentenaire que le monde a vécue depuis la Révolution française, l'histoire d'une co-création de l'apriorisme et de l'empirisme. ${ }^{79}$

Tout compte fait, même si le Contrat social pouvait être un livre pernicieux dans la mesure où il pourrait procurer aux tyrans et aux pseudo républicains un langage de justification qui légitime leur autorité politique au delà des limites circonscrites par les lois, on ne devrait pas oublier pour autant que la «souveraineté du peuple » est un principe qui ne peut être contesté, que la pensée de Rousseau reste avant tout une théorie de la souveraineté qui serait une philosophie critique du pouvoir. Il faut admettre qu'en Chine où le peuple n'a jamais été jusqu'ici la source de la souveraineté et où le régime actuel tente de substituer à la question de légitimité politique celle d'efficacité économique et de stabilité sociale, la théorie de la souveraineté du peuple de Rousseau reste toujours d'actualité.

\section{Le projet institutionnel de Wang Lixiong}

S'il est difficile de mesurer l'impact exact du Contrat social dans la formation de la conscience citoyenne des Chinois, il est néanmoins possible d'éclaircir la manière dont l'esprit du Contrat social inspire les réflexions que mènent les intellectuels chinois d'aujourd'hui sur le projet institutionnel. Il faut pour cela quitter le terrain de la critique textuelle pour entrer dans le monde des idées. Le travail le plus original et le plus audacieux nous semble revenir à Wang Lixiong 王力雄. 80 Bien que ni le terme de "contrat social», ni le nom de Rousseau ne soient mentionnés dans les ouvrages présentant les idées de Wang, certains traits caractéristiques de son projet correspondent pourtant aux grandes thèses proposées par le philosophe français.

Le projet institutionnel de Wang Lixiong se donne pour objectif et pour ambition de réaliser en Chine l'idée centrale de Rousseau: la souveraineté appartient au peuple qui doit être la source du pouvoir. Le système d'élections successives aux multiniveaux qu'il convient d'appeler démocratie progressive est de concevoir l'organisation et l'exercice du pouvoir politique à chaque niveau de l'Etat comme étant le produit d'une volonté et d'un contrôle permanent du peuple. Prenons par

\footnotetext{
${ }^{79} \mathrm{Zhu}$ Xueqin, p. 275.

${ }^{80}$ Deux ouvrages majeurs révèlent son projet institutionnel : le premier est publié au Canada en 1998 par Mirror Books Ltd sous le titre de Rongjie quanli-zhuceng dixuanzhi, 溶解權力——逐層遞選制 [Dissoudre le pouvoir - Le système d'élections successives aux multi-niveaux] ; le second est paru en 2004 chez Hong Kong Press For Social Sciences Ltd, il s'intitule Dijin minzhu - Zhongguo de disantiao daolu 遞進民 主一一中國的第三條道路 [La démocratie progressive - La troisième voie pour la Chine].
} 
exemple la population rurale qui représente $70 \%$ de la population chinoise. Dans un village de base, chaque famille désigne un membre comme représentant. Les représentants des familles forment ainsi le Conseil du village de base et élisent parmi eux un directeur. Les directeurs élus à ce niveau forment ensuite le Conseil de la commune et élisent un directeur à leur tour. Les directeurs des Conseils de commune forment le Conseil cantonal et élisent le directeur de cette instance. De la même façon, se forment le Conseil du district et le Conseil de la province. Au niveau national, ce sont les directeurs des trente et un Conseils de provinces, de régions autonomes et de municipalités relevant directement de l'autorité centrale qui élisent le Président du Conseil national, chef de l'Etat chinois. Le principe est toujours le même: le directeur élu de son Conseil devient membre électeur du Conseil supérieur, la charge d'exécuter les décisions collectives revient au directeur $\mathrm{du}$ Conseil. On peut donc qualifier "la démocratie progressive», d'un système d'élections échelonnées de bas en haut, du local au national, évitant ainsi les risques de la démocratie à grande échelle jugée trop dangereuse pour un pays comme la Chine. A la différence du Contrat social, La démocratie progressive n'est pas un traité de droit politique, mais un traité de méthode d'élections donnant naissance aux institutions politiques nouvelles.

Un autre point qui rapproche le projet institutionnel de Wang Lixiong du Contrat social est l'étroite parenté entre «la volonté générale (terme de Rousseau) et «la volonté sociale » (terme de Wang Lixiong). La volonté générale, selon la définition rousseauiste, "ne regarde qu'à l'intérêt commun». ${ }^{81}$ En ôtant des volontés particulières «les plus et les moins qui s'entre-détruisent, reste pour somme des différences la volonté générale ». ${ }^{82}$ Wang Lixiong transforme « la volonté générale » en volonté sociale en y introduisant la notion de vecteur:

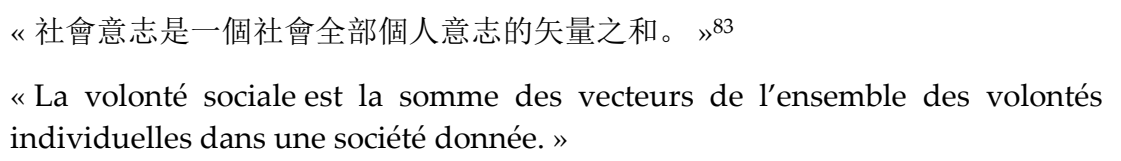

Par rapport au nombre, le vecteur est un élément qui a une direction, ou une orientation. Chaque volonté particulière (ou individuelle) est un vecteur. Autrement dit, les volontés particulières ne diffèrent pas qu'en nombre, elles diffèrent aussi en direction. Si la volonté de tous n'est qu'une somme de volontés particulières, la somme vectorielle de volontés particulières devient la volonté

\footnotetext{
${ }^{81}$ Rousseau, Du Contrat social, p. 50.

${ }^{82}$ Rousseau, p. 50.

${ }^{83}$ Wang Lixiong, Rongiie quanli-zhuceng dixuanzhi, p. 98.
} 
sociale. Il semble que Rousseau a lui-même établi le rapport d'affinité entre volonté générale et volonté sociale:

"C'est ce qu'il y a de commun dans ces différents intérêts qui forme le lien social, et s'il n'y avait pas quelque point dans lequel tous les intérêts s'accordent, la société ne saurait exister. Or comme la volonté tend toujours au bien de l'être qui veut, que la volonté particulière a toujours pour objet l'intérêt privé, et la volonté générale l'intérêt commun, il s'ensuit que cette dernière est, ou doit être seule le vrai mobile du corps social. $»^{84}$

La différence qu'il peut y avoir entre volonté générale et volonté sociale réside dans le fait que la première recouvre ce qui est commun à toutes les volontés particulières comme une sorte de consensus, tandis que la seconde se présente comme un compromis dans un esprit éclectique (voir les deux figures ci-après).

Dans la Figure 1, les cercles A, B, C représentent trois volontés particulières, l'espace D, ayant pour caractéristique d'être commun aux trois cercles, incarne la volonté générale. La partie commune est d'autant plus grande que les trois cercles se superposent davantage. A l'inverse, elle se rétrécit dès que la superposition diminue. La recherche de la volonté générale consiste à agrandir la superposition des volontés particulières.

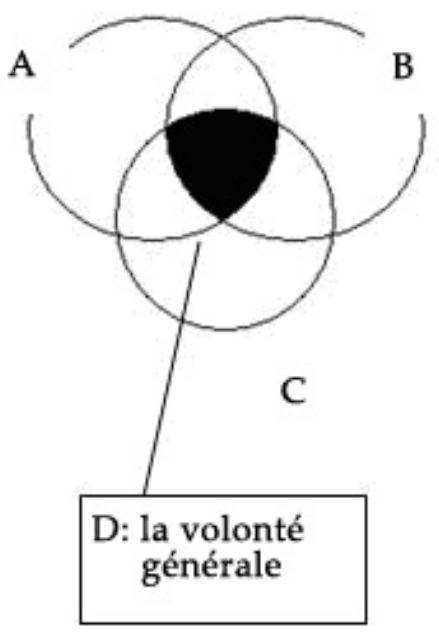

Fig. 1

${ }^{84}$ Rousseau, Du contrat social ou essai sur la forme de la République (Première version, Manuscrit de Genève), in CEuvres complètes, tome III, Pléiade, Paris, Gallimard, 2003, p. 295. 
Dans la Figure 2, les segments A et B signifient deux volontés individuelles (vecteurs), d'orientation différente. Le segment $C$, comme la diagonale d'un parallélogramme, désigne la somme des vecteurs, appelée la volonté sociale. Le schéma peut bien sûr varier en fonction des directions variables des vecteurs. Il s'agit de l'élaboration d'un processus de négociation toujours ouvert de compromis par le dialogue, la concertation, la persuasion et le marchandage.

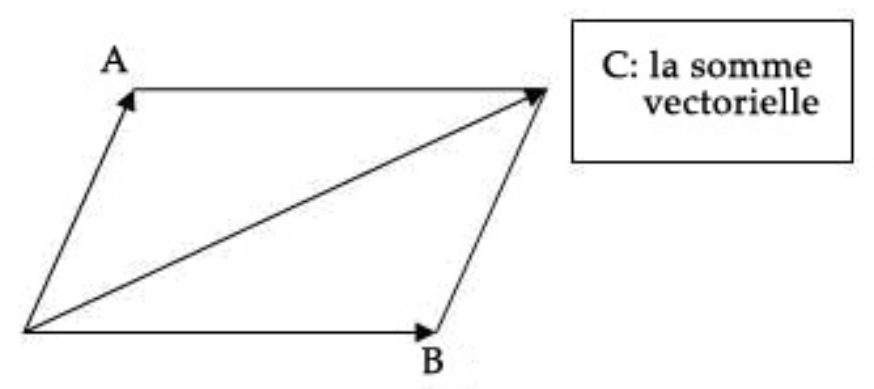

Fig. 2

Trouvant peut-être la présentation trop mécanique et simpliste, Wang Lixiong recourt à l'ancienne métaphore de la langue classique, minxin 民心 (littéralement «le cœur du peuple» qu'il convient de traduire ici par aspirations ou volonté du peuple), pour expliquer ce qu'il entend par la volonté sociale. Il conclut que le meilleur régime politique possible est celui qui sait écouter «le cœur du peuple», c'est-à-dire, respecter constamment la volonté sociale. ${ }^{85}$

Nous savons que Rousseau distingue la volonté générale de la volonté de tous, celle-ci ne regarde que l'intérêt privé et «n'est qu'une somme de volontés particulières». Cette distinction conduit Rousseau à s'opposer à la politique partisane. Il prend comme exemples de volontés particulières les associations (les partis politiques) qui suivent leur intérêt particulier, et non pas l'intérêt commun :

«Enfin quand une de ces associations est si grande qu'elle l'emporte sur toutes les autres... alors il n'y a plus de volonté générale, et l'avis qui l'emporte n'est qu'un avis particulier. $^{86}$

$[\ldots]$

${ }^{85}$ Wang Lixiong, Rongjie quanli-zhuceng dixuanzhi, p. 102 et p. 113.

86Rousseau, Du Contrat social, p. 51. 
Il importe donc pour avoir bien l'énoncé de la volonté générale qu'il n'y ait pas de société partielle dans l'Etat et que chaque citoyen n'opine que d'après lui. $»^{87}$

La démocratie progressive que propose Wang Lixiong n'est pas favorable non plus à la politique des partis. D'abord elle n'a pas besoin des partis politiques pour fonctionner, étant donné qu'elle n'est pas fondée sur le multipartisme comme nous le voyons dans la quasi-totalité des pays dits démocratiques. Ensuite elle ne dissimule pas sa visée de libérer progressivement l'administration chinoise du contrôle du PCC en commençant par le niveau primaire. Tout membre des partis politiques pourra se présenter aux élections en tant que représentant de sa famille. Les élus qui constituent le Conseil du village naturel élisent son directeur en fonction des mérites des candidats et sur leur programme sans tenir compte de la consigne des partis politiques, et les élections devront se passer de la même façon aux niveaux supérieurs. C'est ainsi qu'il espère entamer un processus de « déidéologisation » de l'administration chinoise, de sorte qu'elle observe la neutralité. ${ }^{88}$

Un autre point commun à Rousseau et Wang Lixiong est la non séparation des pouvoirs. Pour Rousseau, la souveraineté, étant l'exercice de la volonté générale, ne peut pas être partagée, d'où son hostilité (ou plutôt sa réticence) à toute séparation des pouvoirs (mises à part ses contradictions à cet égard). Dans le projet institutionnel de Wang Lixiong, le Conseil de chaque niveau exerce à la fois le pouvoir législatif et le pouvoir exécutif. Quant au pouvoir juridique, il est confié aux juges et aux procureurs élus par le Conseil du niveau correspondant pour une majorité des deux tiers, ce qui rend la juridiction responsable devant le Conseil et non vis-à-vis d'une personne, et assure une relative indépendance de la justice.

L'une des singularités de la démocratie progressive, c'est de ne pas fixer de dates pour les élections et de laisser le conseil de chaque niveau en décider librement, comme Rousseau qui prévoyait dans $\mathrm{Du}$ Contrat social l'ouverture des assemblées sans "convocation formelle » préalable pour se tenir et changer l'administration. ${ }^{89} \mathrm{Il}$ faut donc que le peuple puisse se réunir aussi souvent qu'il le souhaite afin de remédier à la corruption ou à la dégénérescence des gouvernements.

Wang Lixiong souligne que la théorie de la démocratie occidentale est paradoxale en ce qu'elle approuve le suffrage universel (direct) et renie le suffrage indirect, mais elle adopte, dans la plupart des cas, la démocratie représentative (indirecte)

${ }^{87}$ Rousseau, Du Contrat social, p. 51.

${ }^{88}$ Wang Lixiong, Dijin minzhu - Zhongguo de disantiao daolu, pp. 212-215.

${ }^{89}$ Rousseau, Du Contrat social, pp. 111-112. 
aux dépens de la démocratie directe. $9^{90}$ En effet, il en va de même pour beaucoup d'hommes et de femmes qui, dans les pays démocratiques, reconnaissent le caractère inévitable de la démocratie représentative, mais éprouvent en même temps quelque nostalgie de la démocratie directe dont ils rêvent de se rapprocher. L'une des visées du projet institutionnel de Wang Lixiong est justement de chercher, par la démocratie progressive, une façon particulière de marier la démocratie directe et la démocratie indirecte, la démocratie participative et la démocratie représentative. Contrairement au suffrage universel tel qu'il s'exerce dans les pays dits démocratiques où les électeurs sont appelés à voter en dehors de leurs expériences personnelles, le système d'élections successives aux multi-niveaux repose sur «un cadre d'expériences » où les votants vivent et travaillent ensemble, communiquant les uns avec les autres sans intermédiaire, tout comme Rousseau aurait souhaité une petite société, un petit Etat où tous les gens se connaissent entre eux. ${ }^{91}$ Wang Lixiong s'autorise à espérer qu'à partir de cette petite société autonome et autogérée se construira un système de l'autonomie progressive débouchant sur un fédéralisme progressif, du local au national (en apportant des solutions nouvelles aux problèmes du Tibet, du Xinjiang et de Taiwan), du niveau national à l'échelle internationale dans une visée du gouvernement planétaire.

Le projet institutionnel de Wang Lixiong peut être perçu comme une tentative de se réapproprier la liberté d'instituer niée ou travestie en Chine depuis deux ou trois millénaires. Tout comme Jean-François Billeter, dans la lignée des thèses de Cornelius Castoriadis, l'affirme dans son remarquable essai Chine trois fois muette:

« Toute société humaine est un système imaginairement institué pour faire face à des conditions nouvelles, puis adapté tant bien que mal à leur évolution ultérieure. Cette liberté d'instituer, qui lui est essentielle, l'homme peut à nouveau en faire un usage audacieux quand les circonstances s'y prêtent. ${ }^{92}$

Cent ans auparavant, Liang Qichao avait prédit dans son fameux article sur Rousseau:

《案盧氏此論 ...... 雖未有行之者, 然將來必徧於大地, 無可疑也。我中國数千 年生息於專制政體之下, 雖然, 民間自治之風最盛焉。誠能博採文明各國地方 之制, 省省府府, 州州縣縣, 鄉鄉市市, 各為團體, 因其地宜以立法律, 從其

\footnotetext{
${ }^{90}$ Wang Lixiong, Dijin minzhu - Zhongguo de disantiao daolu, p. 185.

${ }^{91}$ Wang Lixiong, p. 156.

${ }^{92}$ Jean-François Billeter, Chine trois fois muette, Paris, Editions Allia, 2000, p. 145. Billeter s'inspire ici directement de l'ouvrage central de la pensée de Corneluis Castoriadis intitulé L'institution imaginaire de la société, Paris, Seuil, 1975.
} 
民欲以施政令, 則成就一盧梭心目中所想望之國家, 其路為最近, 而其事為最 易焉。果爾, 則吾中國之政體, 行將為萬國師矣。»93

« L'idée de Rousseau ... deviendra certainement réalité partout dans le monde, bien qu'il n'y ait pas encore eu d'exemple aujourd'hui. La Chine vit depuis des millénaires sous le régime autoritaire, malgré une forte tradition d'autonomie populaire. Si elle peut s'inspirer des institutions locales des pays civilisés, faire en sorte que chaque province, chaque préfecture, chaque sous-préfecture, chaque district, chaque canton, chaque ville, constituent un corps à leur niveau, établissent des lois qui leur conviennent au mieux, gouvernent selon la volonté de leur population, alors elle créerait, par le chemin le plus court et de la façon la plus commode, un Etat dont rêvait Rousseau. Si une telle entreprise se réalise un jour, l'institution de la Chine servirait d'exemple à tous les pays. »

Cent ans plus tard, " comme de longs échos qui de loin se confondent », l'appel de Liang Qichao et le projet institutionnel de Wang Lixiong se répondent à travers la problématique de l'œuvre de Rousseau. ${ }^{94}$

${ }^{93}$ Liang Qichao, « Lusuo xuan'an », p. 110.

${ }^{94}$ Charles Baudelaire, Les Fleurs du mal, in CEuvres complètes, tome I, Pléiade, Paris, Gallimard, p. 11. 